\title{
Search for Galactic cosmic ray sources: The multimessenger approach
}

\author{
Julia Becker Tjus ${ }^{\mathrm{a}}$ \\ Theoretische Physik IV: Plasma-Astroteilchenphysik, Ruhr-Universität Bochum, 44780 Bochum, Germany
}

\begin{abstract}
The search for the sources of high-energy cosmic rays (CRs) has made significant progress the past decade. By including multimessenger methods, the general picture of the presence of a Galactic component at low energies and an extragalactic one at the highest energies has been strengthened. Yet, unambiguous proof of the exact origins of CRs is missing. In this review, the current scientific status on Galactic CR sources from theory and experimental data is summarized. In particular, the focus of this review lies on the search for photon and neutrino signals from the Galaxy and their theoretical interpretation in the context of the quest for the origin of high-energy cosmic rays. The use of multiwavelength data, from radio to $\mathrm{TeV}$ energies, as well as the option of coincident observations of different wavelength bands in order to pin-point the sources of Galactic CRs are discussed. Finally, the objectives for the field of astroparticles to reach the goal of unambiguously identifying Galactic cosmic ray sources within the next decades are presented.
\end{abstract}

\section{Introduction}

During recent years, the search for the sources of highenergy cosmic rays (CRs) has made large progress. By including multimessenger methods, the general picture of the presence of a Galactic component at low energies and an extragalactic one at the highest energies has been strengthened. Yet, unambiguous proof of the exact origins of CRs is still missing. Figure 1 shows the observed CR spectrum, spanning more than 11 orders of magnitudes in energy. Basic and well-known features of the all particle spectrum are a power-law behavior with the two prominent breaks, usually referred to as the knee at around $10^{6} \mathrm{GeV}$ and the ankle at around $10^{9.5} \mathrm{GeV}$. The spectrum finally cuts off at around $10^{10.5} \mathrm{GeV}$ as it was confirmed in recent years [1]. Detailed information on the composition of this spectrum of charged nuclei is now provided by experiments from the lowest to the highest energies. While the energy spectrum itself is quite well measured, the concrete sources could not be pin-pointed yet due to the diffusion of CRs through Galactic (and extragalactic) magnetic fields.

The multimessenger approach in the search for the origins of CRs builds on including all available information from charged CRs as well as from neutral secondaries that are produced near the sources through hadronic interactions. In particular, neutrino and $\gamma$-ray fluxes arise in the following interactions of cosmic rays (CRs) with matter $\left(p_{\text {target }}\right)$ or photon fields $\left(\gamma_{\text {target }}\right)$ :

$$
C R+p_{\text {target }} \rightarrow \#\left(\pi^{ \pm, 0}\right)+X
$$

\footnotetext{
a e-mail: julia.tjus@rub.de
}

$$
\begin{aligned}
C R+\gamma_{\text {target }} & \rightarrow\left\{\begin{array}{l}
\Delta^{+} \rightarrow\left\{\begin{array}{l}
p \pi^{0} \\
n \pi^{+}
\end{array}\right. \\
\#\left(\pi^{ \pm, 0}\right)+X
\end{array}\right. \\
\pi^{ \pm} & \rightarrow \mu+v_{\mu} \rightarrow\left(e+v_{e}+v_{\mu}\right)+v_{\mu} \\
\pi^{0} & \rightarrow \gamma \gamma .
\end{aligned}
$$

The final, neutral particles, neutrinos $v$ and photons $\gamma$ carry approximately $5 \%$, respectively $10 \%$ of the original CR energy and point back to their sources directly. The detection of the different particle species have different advantages and disadvantages as summarized in Table 1 and as discussed in detail below. In this paper, the focus lies on presenting the state of the art concerning multimessenger searches for Galactic CRs. Central arguments for supernova remnants (SNRs) as the main contributing source class will be presented (Sect. 2), followed by the summary of current and future approaches to verify or falsify this hypothesis with multimessenger data (Sect. 3). The paper concludes with an outlook for the prospects of identifying the concrete sources of Galactic CRs in the next decades (Sect. 4).

\section{Observations and theory: SNRs as the most reasonable $C R$ candidates}

Despite the fact that charged CRs are not revealing directional information directly, their latest measurements deliver larger pieces of information on the nature of their sources and they are reviewed below.

Energy budget. As first suggested in [2], supernova remnants (SNRs) can provide the observed energy budget up to the knee if on average $10 \%$ of the total energy of

This is an Open Access article distributed under the terms of the Creative Commons Attribution License 4.0, which permits unrestricted use, distribution, and reproduction in any medium, provided the original work is properly cited. 


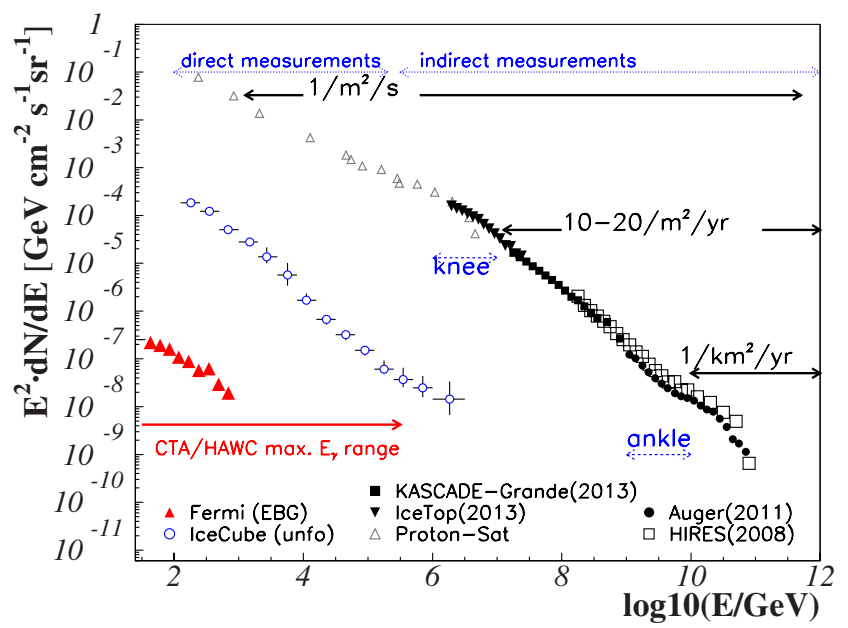

Figure 1. All particle CR energy spectrum from $100 \mathrm{GeV}$ up to $>10^{11} \mathrm{GeV}$. In addition, the diffuse extragalactic $\gamma$-ray background and the diffuse unfolded neutrino spectrum, composed of atmospheric neutrinos at low energies and astrophysical ones at high energies, are shown. The flattening of the neutrino spectrum towards high energies show the transition from atmospheric to astrophysical neutrinos. $\gamma$-rays and neutrinos carry $\sim 10 \%$ resp. $5 \%$ of the primary CR energy. While neutrino measurements cover the knee range already today, $\gamma$-ray instruments will start reaching it within the next years (see indicated energy range for CTA).

Table 1. Pieces of information available from CRs, $\gamma$-rays and neutrinos. $\left(E_{\mathrm{min}}^{\mathrm{CR}} ; E_{\mathrm{max}}^{\mathrm{CR}}\right)_{2015}$ gives the cosmic ray energy range that can be detected by today's generation telescopes. Here, the actual energy range covered by photon and neutrino telescopes is translated into $\mathrm{CR}$ energies by assuming that primary CRs carry a factor $\sim 20(\sim 10)$ more energy than the neutrinos (photons). The CR energy range expected to be covered by next generation's telescopes, $\left(E_{\min } ; E_{\max }\right)_{\text {next-gen }}$, is summarized in Column 3 .

\begin{tabular}{l|lllll}
\hline & $\left(E_{\min }^{\mathrm{CR}} ; E_{\max }^{\mathrm{CR}}\right)_{2015}$ & $\left(E_{\min }^{\mathrm{CR}} ; E_{\max }^{\mathrm{CR}}\right)_{\text {next-gen }}$ & $\begin{array}{l}\text { individual } \\
\text { sources }\end{array}$ & $\begin{array}{l}\text { diffuse } \\
\text { signal }\end{array}$ & $\begin{array}{l}\text { leptonic } \\
\text { pollution? }\end{array}$ \\
\hline $\mathrm{GeV}]$ & $\left(<1 ;>10^{11}\right)$ & $\left(<1 ;>10^{11}\right)$ & difficult & yes & no \\
$\gamma_{\mathrm{S}}$ & $\left(>1 ;>10^{5}\right)$ & $\left(>1 ;>10^{6}\right)$ & yes & yes & yes \\
$\nu \mathrm{S}$ & $\left(2 \cdot 10^{5} ;>2 \cdot 10^{7}\right)$ & $\left(2 \cdot 10^{5} ;>2 \cdot 10^{8}\right)$ & expected & yes & no \\
\hline
\end{tabular}

each supernova explosion is converted to the acceleration of CRs, see e.g. [3]. As a back-of-the envelop estimate, the CR luminosity $L_{\mathrm{CR}}$ can be calculated from the differential energy spectrum measured at Earth, $d N / d E$,

$$
L_{\mathrm{CR}}=\int_{E_{\min }}^{E_{\max }} \int v_{\mathrm{CR}} /(4 \pi) \cdot \tau \cdot d N / d E \cdot E d E d V .
$$

Here, $\tau$ is the lifetime of CRs in the emission volume, $v_{\mathrm{CR}}$ is the CR's velocity, $v_{\mathrm{CR}} \approx c$ and $V$ is the emission volume. Assuming a Galactic origin of CRs below the knee, i.e. $E<10^{15} \mathrm{eV}$, the $\mathrm{CR}$ luminosity can be calculated to approximately $L_{\mathrm{CR} \text {, knee }} \sim 10^{41} \mathrm{erg} / \mathrm{s}$, where the Galaxy is assumed as the emission volume, $V=$ $V_{\text {gal }}$ and the CR lifetime corresponds to the average time it takes CRs to escape the Galaxy, $\tau=\tau_{\text {esc }}$. In its simplicity, this estimate rather represents an order-ofmagnitude prediction than a detailed description. It is in any case a requirement for a source class to be able to provide an energy budget of this order, but it should be enough if the criterion is matched within a factor 3 [3]. One of the most promising source classes fulfilling this luminosity criterion is the one of supernova remnants. Given that in the explosion of a star into the interstellar medium, an energy of about $E_{\mathrm{SN}} \sim 10^{51} \mathrm{erg}$ is released and that the explosion rate in the Galaxy is approximately $\dot{n} \sim 0.02 / \mathrm{yr}[4]$, the CR energy budget measured at Earth can be reproduced with a fraction of $\eta \sim 0.1$ of the supernova's energy budget going into CRs:

$$
L_{\mathrm{CR}} \approx 2 \cdot 10^{41} \mathrm{erg} / \mathrm{s} \cdot\left(\frac{\eta}{0.1}\right) \cdot\left(\frac{\dot{n}}{0.02 \mathrm{yr}^{-1}}\right) \cdot\left(\frac{E_{\mathrm{SN}}}{10^{51} \mathrm{erg}}\right) .
$$

Other potential CR accelerators like pulsars and energetic binary systems have difficulties achieving this large energy budget, see Fig. 2.

It can be shown that extragalactic sources are too faint to explain the low-energy part of the spectrum. Arguments concerning the maximum energy of the sources as well as the high level of isotropy do not really allow for Galactic sources to accelerate to beyond the ankle. This luminosity criterion is one of the most striking pieces of evidence that the CR spectrum below the knee is of Galactic origin, while the part above the ankle must be of extragalactic origin. Where the transition between Galactic and extragalactic CRs happens exactly is not clear yet and poses one of the most important questions for the next decades in astroparticle physics.

Spectral behavior and composition. The generally observed power-law spectral behavior can be explained by the concept of diffuse shock acceleration at the SNR shock front, with the first idea presented in [6,7] and further developed by e.g. [8,9]. While SNRs explain the 


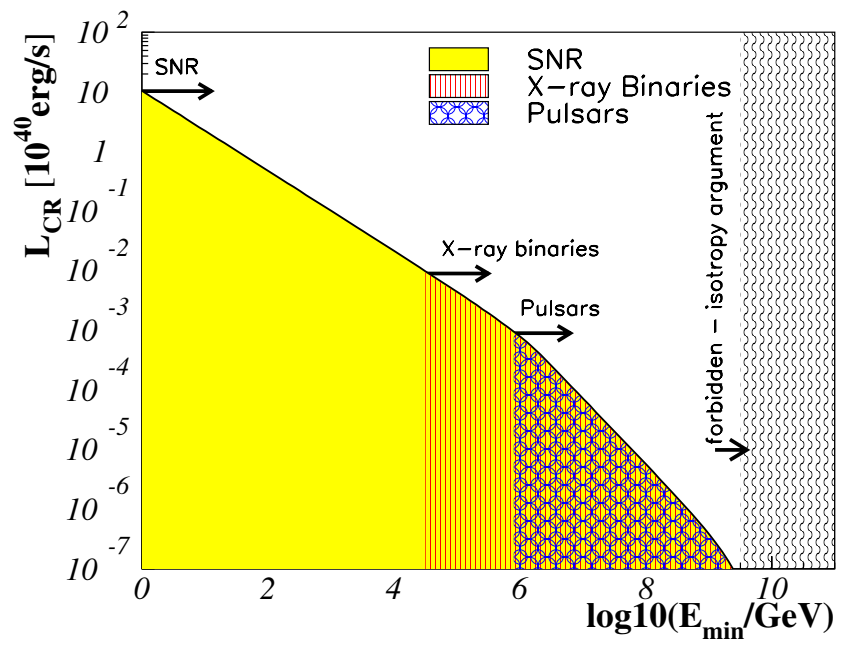

Figure 2. CR luminosity calculated following Eq. (5), dependent on the minimum energy $E_{\min }$. The shaded areas indicate which source class can explain the energy budget, starting from a certain fixed energy $E_{\min }$. It is indicated that SNRs can explain the entire budget from $1 \mathrm{GeV}$, while contributions at higher energies could arise from X-ray binaries (XRBs) or pulsars. Figure modified from [5].

CR energy budget quite well, they do not reach knee energies naturally as the average magnetic field strength of around $10 \mu \mathrm{Gauss}$ is not enough to reach $10^{15} \mathrm{eV}$. This conundrum can be solved by including magnetic field amplification as suggested in [10], where the CR pressure gradient at the shock front can generate magnetic fields significantly higher than in the interstellar medium, up to a few $100 \mu$ Gauss. This concept is supported by observations as reviewed in [11]. However, even for young SNRs like Tycho which are expected to reach the highest energies, calculations including field amplification only provide a maximum energy of $\sim 10^{5} \mathrm{GeV}$ [12]. These expand into a thin medium, while fast expansion into a dense wind would be able to go towards higher energies [13-15].

The spectral shape of the CR energy spectrum is relatively well-measured from the lowest to highest energies. In particular, recent measurements provide information on individual nuclei spectra up to iron. Direct measurements for proton to iron nuclei spectra, performed by balloon and satellite experiments, reach up to several $\mathrm{TeV}[16,17]$. It turns out that the hydrogen energy spectrum is steeper than the one for heavier nuclei [16]. PAMELA [18] and CREAM [16] data also show a break in the hydrogen and helium spectra, which could, however, not be confirmed by the newest AMS-02 results $[17,19]$. These discrepancies need to be resolved with future data. Heavier nuclei show a similar hardening of the spectrum at around a few $100 \mathrm{GeV}$ energy per nucleon [16] from $E^{-2.77}$ to $E^{-2.56}$. Such a break in the spectrum would be expected if there is a nearby source contributing to the spectrum, e.g. [20]. Alternatively, the scattering centers for acceleration in SNRs can be produced by CRs themselves via the streaming instability [21]. The spectral behavior can also be explained by the contribution of the polar cap component of SNRs from heavy progenitor stars, in which the magnetic field is parallel to the SNR shock front, giving rise to a second and flatter component towards higher energies [14].

At higher energies, in the energy region between the knee and the ankle, where only indirect measurements of the composition via the analysis of CR air showers is possible, first results on the energy-dependent composition comes from the KASCADE-Grande experiment [22,23]. The light component seems to flatten at around $10^{17} \mathrm{eV}$, possibly indicating the rise of an extragalactic component. The KASCADE-Grande results also indicate that the heavy component continues as a power-law up to around $10^{18} \mathrm{eV}$, where it is estimated to contribute about the same amount as the light component. It should be noted that individual and concrete nuclei spectra are extremely difficult to derive via this indirect detection method and systematic uncertainties are generally larger compared to direct detection. The continuation of the iron spectra up to $10^{18} \mathrm{eV}$ in combination with the proton data can be interpreted as a superposition of cutoffs that are dependent on the nuclei's charge $Z$, which is in accordance with the hypothesis of a cutoff of the acceleration process of Galactic sources, see e.g. [24], and also matches scenarios of CR acceleration in SNRs with heavy progenitor stars $[13,14]$. The extragalactic scenario would in this case take over at around $10^{18} \mathrm{eV}$ [25], but would imply a relatively heavy composition at the ankle, which is not seen in the data of ultra high energy CR experiments Auger and Telescope array [26,27]. In addition, in such a scenario, a proper, statistical fit to the data from $\mathrm{GeV}$ to $10^{11} \mathrm{GeV}$ energies requires a third component, contributing in the knee-to-ankle region [28], in particular to explain sub-structures as the second knee at a few $10^{17} \mathrm{eV}$. An alternative scenario is that the extragalactic component becomes dominant around $10^{17} \mathrm{eV}$, with a large contribution from protons that lost energy via the Bethe-Heitler pair production process [29]. IceTop, the surface CR detector integrated in the IceCube experiment, recently presented first results on the composition in the knee region. The comparison of KASCADE Grande data with IceTop results as well as upcoming data from the HiSCORE experiment, sensitive in a similar energy range [30], will help to understand systematics and narrow down the composition in this interesting energy region.

Anisotropy. The detected CR spectrum is distributed isotropically in first order approximation. At a sub-percent level, however, a dipole anisotropy is observed in the $\mathrm{TeV}$ range, see e.g. [31] for a summary of the experimental results. Sub-structures at higher multipoles are present as well at a lower intensity level. At PeV energies, the dipole existing at $\mathrm{TeV}$ energies fades and a larger deficit shows instead. An energy dependent explanation of the dipole structure is therefore needed. When taking into account the enhanced density of CR sources in the Galactic Center, the dipole anisotropy level is actually too low and standard simulations overestimate the anisotropy by an order of magnitude [21,32]. One solution to this problem is presented in [33] in a phenomenological model where the level of turbulence follows the source density. There 
are other scenarios to explain such an anisotropy, like the presence of a local flow in the interstellar medium [34]. The higher multipole structure can be explained by the magnetic field influence of the heliotail [35], local turbulence [36] or nearly extinct pulsar tails [e.g. 37]. It is of high importance to consistently be able to explain the anisotropy structure when modeling the CR transport to show that the global model is consistent with observations.

Electrons and positrons. The measurement of electrons and positrons contribute to the knowledge on hadronic CRs: the flux measured at Earth must certainly arise from nearby sources due to strong synchrotron losses. Electrons are expected to be directly accelerated in SNRs and help to understand the basic acceleration mechanism at the accelerator [e.g. 13]. Positrons are produced in $\mathrm{CR}$ interactions [e.g. 38]. Both electron and positron spectra show a hardening of the components [39]. A dark matter interpretation of these is possible [e.g. 40], but the observed features can also be explained within classical astrophysical scenarios [e.g. 13,41].

Pitfalls for the "standard model". On the one hand, the presented pieces of information partially work quite well for SNRs as the dominant sources of Galactic CRs. On the other hand, there are still many open questions. In the standard scenario for the calculation of energy budget and spectral behavior, for example, all SNRs are believed to follow the same spectral behavior and provide the same energy budget. It is known from theory and from gamma-ray data that this is far from reality. CR spectra from SNRs evolve with time and are expected to reach their maximum energy at the earliest stage, most likely even before they reach the Sedov-Taylor phase. With increasing age, the maximum energy of the CR spectrum is expected to decrease. Gamma-ray observations show that the spectral behavior of the source depends on the SNR as well: old SNRs in dense environments have steep gammaray spectra, indicating steep CR spectra, while young SNRs in the thin ISM have flat spectra. Thus, the observed CR spectrum is expected to be a mixture of different spectral indices and maximum energies. Also, while the concept of magnetic field amplification gets supernova remnants to accelerate up to the knee, it is certainly a challenge to reach to even higher energies. Explosions into dense environments is an option, although gammaray measurements seem to reveal quite steep spectra under these conditions. The high level of isotropy in the TeV-PeV energy range is not explained naturally either, as a standard distribution of SNRs would result in a higher anisotropy as compared to what is observed. Finally, it is not trivial to explain the obsered electron and positron spectra, as they typically require an average electron spectral index for SNRs that is significantly steeper than $E^{-2.3}$, which is what is observed from electron synchrotron radiation at radio wavelengths. The following sections will mostly focus on the first and primary goal: how can the multimessenger approach be used to unambiguously identify the sources of Galactic CRs? This is not completely disentangled from the open questions above, but in order to have a first identification of sources, it is not necessary to answer all detailed physics questions. This needs to be done in a second step after solid prove of where to look for the concrete sources.

\section{Photons and neutrinos: Pin-pointing Galactic CR sources}

\subsection{The multiwavelength electromagnetic spectrum}

Gamma-ray astronomy had its beginning in the 1960s, when the satellite Explorer XI measured the first 22 photons above $50 \mathrm{MeV}$ in energy [42]. Since then, spacebased $\gamma$-ray astronomy has made major advances, mainly with the launch of the successful experiments EGRET in the 1990s and Fermi in 2008. Today, more than 3,000 sources are known and both the Galactic and extragalactic background are measured in detail up to $>100 \mathrm{GeV}$ in energy. Earth-based $\gamma$-ray astronomy started with the installation of Imaging Air Cherenkov Telescopes (IACTs) in the 1980s and with the first generation telescopes, more than 10 sources could be identified at $\mathrm{TeV}$ energies, see e.g. $[43,44]$ for recent reviews of the field. Incredible advances have been made with the installation of the second generation telescopes H.E.S.S., MAGIC and VERITAS. Within 10 years time, the number of sources in the $\mathrm{TeV}$ energy range could be increased to more than 150 , providing detailed information on the spectral behavior, variability and spatial extension. Further information comes from the Water Cherenkov Array Milagro, covering the entire sky, but at a lower sensitivity. The measurement of the energy spectra at the highest energies is of crucial importance for the search of the sources of high-energy CRs: Inverse Compton radiation is limited in its maximum energy via the Klein-Nishina effect and bremsstrahlung cuts off early due to the typically loss-limited energy of the primary electron spectra inducing the radiation [45]. Only photons from $\pi^{0}$-decays can reach energies to $100 \mathrm{TeV}$ and above, as the spectrum basically cut off at $\sim 10 \%$ of the primary CR's energy [46].

IACTs have managed to identify more than 20 shelltype SNRs at TeV energies, where about half of these detections are associated with molecular clouds close to the SNR. For the first time, this energy region can be investigated systematically concerning the properties of the origin of the emission. A fit to the spectral energy distribution of 24 SNRs from radio wavelength to $\mathrm{TeV}$ energies was performed in [47], including both IC, bremsstrahlung and $\pi^{0}$-decay. It turns out that 21 of the SNRs are well-fit with a dominant contribution from $\pi^{0}$-decays. The investigation of the primary $\mathrm{CR}$ spectral behavior shows a complex distribution, with some flat sources (close to $E^{-2}-E^{-2.3}$ ), usually represented by young SNRs in the interstellar medium, and several older SNRs in molecular clouds with steep spectra. A striking fact is that the proton spectra are generally steeper than the electron spectra (see Fig. 3). This is rather unexpected. While proton spectra can of course be steepened by local diffusion, the steepening of electron spectra by radiation losses would at least be expected. This statistical sample now provides a first opportunity 


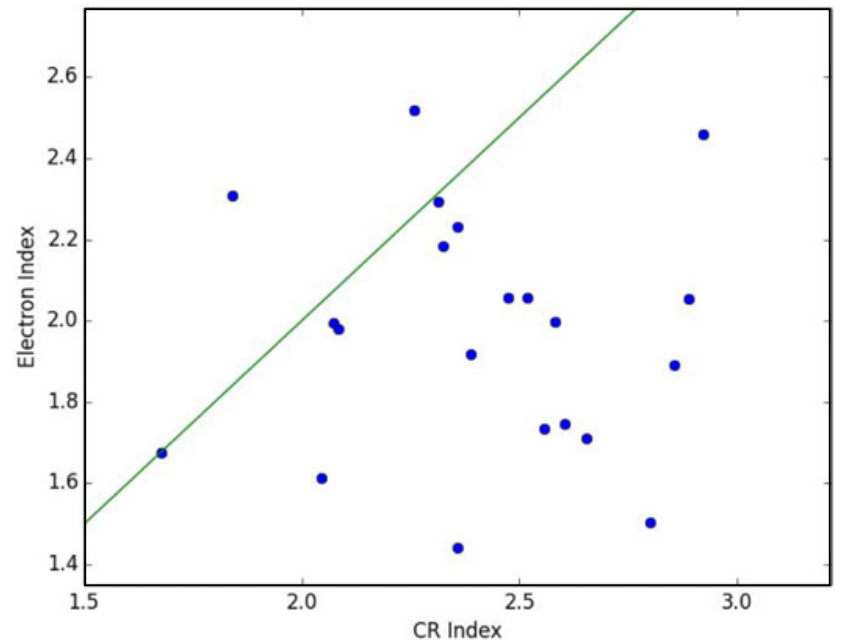

Figure 3. Spectral indices for electrons and for protons, defined differentially, i.e. $d N / d E \propto E^{-\alpha_{i}}$ and $\alpha_{i}=\alpha_{e}, \alpha_{p}$. Values are derived from gamma-ray data, assuming that the dominant contribution to the high-energy part of the SNR SEDs is dominated by hadronic emission. The dashed line indicates equality, $\alpha_{e}=\alpha_{p}$.

to investigate SNRs as potential CR candidates. These 21 sources should represent about $10 \%$ of the total number of emitters, assuming that a supernova explosion rate of $\sim 0.02 \mathrm{SN} /$ year in the Galaxy [4] produces SNRs that are active CR accelerators for about 10,000 years on average. The detected SNRs should then represent a mixture of different SNR stages: theory predicts the evolusion of CR spectra with time, where young SNRs (ages $<1,000$ years) can potentially reach maximum energies up to the knee. With age, this maximum energy decreases. The natural mixture of SNR ages in the Galaxy should therefore represent a distribution of CR spectra that is on average contributing to the observed CR spectrum. These detected CRs will certainly not come from those SNRs that we observe today, as their escape time is longer than the production time in SNRs $\left(t_{\mathrm{esc}} \sim 10^{6}-10^{7}\right.$ years $)$. Thus, while the distribution spectra should be representative, the exact positions of the SNRs are not.

This distribution of spectral indices has direct consequences for the interpretation of supernova remnants as the dominant CR sources: naively, one would expect that, with such a steep distribution, the average CR spectrum should be steeper than the typically assumed $E^{-2.3}$ type behavior. Thus, problems in the reproduction of the spectral behavior of the observed diffuse CR spectrum should be expected. Similar difficulties could arise concerning the normalization of the spectrum: is the number of SNRs observed in gamma-rays really matching the total energy budget of CRs, or do they over- or underproduce the observed spectrum? As more and more precise gamma-ray data come in, these questions will in the future be possible to answer by combining the photon data with the proper propagation of the individual SNR spectra from the source to Earth [48] using numerical tools like GALPROP [49], DRAGON [50] or PICARD [51]. Although the data used in such a statistical analysis are still ambiguous, as they in many cases could also represent an IC- or bremsstrahlungs-dominated spectrum, it is clear that hadronic sources with steep spectra exist. Three of these SNRs - W44, IC443 and most recently $\mathrm{W} 51 \mathrm{C}$ - have been proven to be dominated by the hadronic component, as the low-energy cutoff of the pion-decay was measured by Fermi [52-54]. In the future, a detailed investigation of the spatial distribution of hadronically- and leptonically-induced $\gamma$-ray spectra is necessary to solve the question which of the other remnants is actually dominated by a hadronic signal [55]. Multiwavelength investigations will help to solve this question. In particular, the search for $\mathrm{CR}$ induced ionization signatures will provide further evidence, as pointed out in $[56,57]$. At sub-GeV energies, $\mathrm{CRs}$ ionize the medium and molecular ions are formed subsequently:

$$
\mathrm{CR}+\mathrm{H}_{2} \rightarrow \mathrm{CR}^{\prime}+\mathrm{H}_{2}^{+}+e^{-} \rightarrow \mathrm{H}_{3}^{+} / \mathrm{H}_{2} \mathrm{D}^{+} / \mathrm{HCO}^{+} / \ldots
$$

This production chain creates molecules in an excited state in a way that rotational and vibrational line signatures are expected and can go up to extremely complex molecules. For CR physics, the smalles of these molecules like $\mathrm{H}_{2}^{+}$and $\mathrm{H}_{3}^{+}$are most interesting, as they provide in an excited state in a way that rotational and vibrational line signatures can be detected. $\mathrm{H}_{2}^{+}$quickly reacts to form $\mathrm{H}_{3}^{+}$and has thus never been detected in ionization lines. A source of strong ionization like it is expected by CRs at shock fronts of SNRs could be a good place to look for this direct ionization tracer [56]. $\mathrm{H}_{3}^{+}$signatures have been detected from different sources, in particular also from IC443 using Herschel [58]. The inferred ionization rate is a factor of $\sim 10$ above the Galactic average level. As this remnant is also detected in gamma-rays, there might be a possible connection to $\mathrm{CR}$ acceleration. The search for the larger molecules $\mathrm{CO}, \mathrm{HCO}^{+}$, and $\mathrm{DCO}^{+}$ also indicates enhanced ionization in the vicinity of the $\gamma$-ray emitting SNR W28, possibly correlating with the gamma-ray signal [59]. Here, the connection between the line intensities and the actual ionization rate is not as straight forward as for $\mathrm{H}_{3}^{+}$and further investigations are necessary to confirm this trend. Detailed correlation studies are of course necessary to confirm or exclude such correlations. In particular the radial profiles of $\gamma$-rays and ionization-induced molecular lines can be compared in the future to draw conclusions as it was shown in [60]. It should generally be kept in mind that this approach does not necessarily work by itself, but only in combination with other pieces of evidence for hadronic acceleration: $\mathrm{CR}$ ionization happens below $1 \mathrm{GeV}$, where the concrete energy spectrum of the CR sources cannot be derived from hadronic signatures directly, as the latter only become effective above $\mathrm{GeV}$ energies. The future $\gamma$-ray telescopes CTA [61] and HAWC [62] are therefore crucial building blocks for the identification of the sources of Galactic CRs: $\gamma$-ray energies of $100-300 \mathrm{TeV}$ can be reached with these next generation instruments. This corresponds to CR energies of 1-3 PeV and will give first evidence of where to find the sources of Galactic CRs contributing in the knee region. The improved resolution of $0.02^{\circ}$ of CTA will give the opportunity to study SNR shock fronts with spatial 
resolution. Combining the pieces of information from all the different messengers will finally deliver a full picture and contribute, together with pieces of information from neutrinos and charged CRs, to the first full identification of the sources of the measured CR spectrum.

\subsection{Neutrinos}

Neutrinos have long been discussed as an unambiguous signature for the sources of CRs, see e.g. [5,63,64]. Neutrino telescopes like DUMAND, Baikal, AMANDA and ANTARES have paved the way for a signal detection since the 1990s, see e.g. [5] for a review. Due to the small interaction cross section and the dominant background of atmospheric neutrinos, a $\mathrm{km}^{3}$-sized detector - IceCube - needed to be build for the first detection of a signal of astrophysical high-energy neutrinos $[65,66]$. The final sample contains 37 events detected in three years including an atmospheric background of the order of 15 events. As the distribution of these neutrinos is isotropic in the sky, no point source could be identified so far. This indicates that it must be several sources contributing to the signal and leaves room for the discussion of several source classes to be responsible for such a signal. The events are detected in the neutrino energy range of approximately $10 \mathrm{TeV}$ to $2 \mathrm{PeV}$, corresponding to $\mathrm{CR}$ energies of $200 \mathrm{TeV}$ to $40 \mathrm{PeV}$. Thus, this neutrino flux represents the region around the CR knee and above. The identification of point sources could give first evidence of those sources contributing to the knee to ankle region. The spectral behavior appears to be steeper than $E^{-2}$, with recent measurements pointing towards a spectrum of $E^{-2.4}$ [67] when assuming a single power-law. Higher statistics will give a more precise measurement of the actual spectrum. As the spatial distribution of the detected neutrinos is isotropic, a larger number of events have directions which are not compatible with the Galactic plane. It is therefore likely that at least part of the signal comes from extragalactic sources. This hypothesis is underlined by the fact that the spectrum extends without cutoff up to $2 \mathrm{PeV}$ neutrino energy, indicating sources that reach up to $40 \mathrm{PeV}$ $\mathrm{CR}$ energy. It is also consistent with theoretical expectation that a contribution to the diffuse neutrino flux as measured with IceCube derived from the observation of gamma-ray data can at maximum reach $2-3$ events $[47,68]$. Figure 4 shows expected diffuse fluxes arising from CR interactions in the Galaxy: the contribution from interactions in the ISM is shown as the red, dashed line [69], while the total diffuse flux from neutrinos emitted close to the shock front of the SNRs is shown as the black, solid line [47]. The blue, dot-dashed line represents a recent calculation of the expected contribution from atmospheric neutrinos [70], representing the dominant background for neutrino searches in the northern hemisphere for IceCube, while data show the deviation from this expectation towards higher energies [71]. The figure emphasizes that the detected signal ${ }^{1}$ can at maximum be explained in parts by SNRs. Extragalactic sources have been suggested as the

\footnotetext{
${ }^{1}$ Data shown here are the unfolded muon spectrum, where the astrophysical contribution is visible in the flattening of the spectrum towards higher energies $[71,72]$.
}

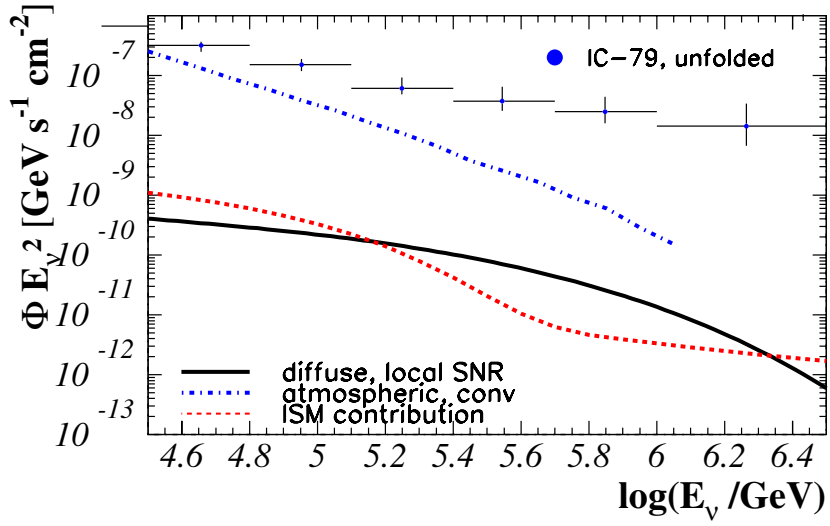

Figure 4. Summary of energy spectra for diffuse contributions from Galactic CRs. For a description of the shown fluxes and data, see text.

dominant source class, in particular emission from active galaxies [73] or starburst galaxies [74].

Concrete searches for point sources and stacked signals are performed with IceCube via the detection of neutrino-induced muons. In contrast to the analysis described above, where all neutrino flavors are included, but applying strong selection criteria, the search for point sources focusses on the large number of muon-neutrinos that interact via charged current interactions - IceCube detects approximately 200 neutrino-induced muons per day, most of which are of atmospheric origin [70]. The muon produced in these neutrino-nucleon interactions points back to the direction of the neutrino with $1^{\circ}$ spatial resolution, while other interaction channels produce cascade signatures where the pointing is of the order of $10^{\circ}$ or worse. The muon channel is therefore very well-suited for point source searches and gives limits to point sources and stacked signals that could already be used in the past to exclude certain source classes as potential neutrino emitters [75]. Latest results for the search for Galactic extended or point sources with IceCube show no evidence for a signal [76]. The Cygnus region is one of the most interesting parts in the Galaxy for IceCube, as it is a star forming region located in the northern hemisphere. Previous data samples partially showed slight, but non-significant excesses of neutrino emission towards that direction, but the full picture of all data, and in particular the newest data, could not confirm any evidence for a signal yet. The search for individual SNRs is still not sentitive enough to detect any of the sources. ANTARES, located in the northern hemisphere, has a very good view on the Galactic Center, on the other hand [77], but with a sensitivity that is still too low to be able to detect a signal. These studies for the sources of Galactic CRs will lead the way for future detections with the next generation telescopes KM3NeT [78] and IceCube-Gen2 [79]. While KM3NeT builds on the experience gained with current generation telecopes like ANTARES [77] in the Mediterranean, IceCube-Gen2 will complement IceCube at the South Pole with an array of about $10 \mathrm{~km}^{3}$. With this and next generation's telescopes, neutrino astronomy will play an important role 
in providing a crucial building block to finding the sources of Galactic CRs and determining their contribution to the detected diffuse signal of astrophysical high-energy neutrinos.

\section{Summary and outlook}

The multimessenger approach has proven itself as an extremely valuable tool to move towards the identification of the sources of Galactic CRs: major progress has been made with charged CRs, photons and neutrinos, summarized here, with an outlook for the potential of development of the field given in the same context:

- Measurements of charged CRs now deliver details on directional information, energy budget, spectral behavior and composition which include constraints to possible source classes of CRs and led to the conclusion that supernova remnants are the primary candidate to make up the dominant part of the CR spectrum below the knee. This does, however, not mean, that this is the final answer. There are still several open questions, like, how do SNRs accelerate up to the knee and beyond? In the future, the investigation of the region between the knee and the ankle of the CR spectrum will become particularly important, as there is the potential to identify the transition between Galactic and extragalactic CRs. By this, the maximum energy of Galactic accelerators can be determined. Interestingly, the first detection of astrophysical neutrinos is in this energy region and future gamma-ray instruments start to reach the region around the knee and even above (see Fig. 1 and Table 1). The upcoming decade(s) therefore seems to have the potential to finally pin-point the sources that are dominantly responsible for the observed CR spectrum.

- The identification of three SNRs as hadronic emitters in gamma-rays is a major step towards the identification of SNRs as CR sources. These detections show that SNRs do accelerate SNRs. What they cannot deliver yet is if SNRs actually do make up the entire $\mathrm{CR}$ energy budget. Also, the three sources have very steep spectra towards $\mathrm{TeV}$ energies and can certainly not explain the spectral behavior of the observed CR spectrum. It is therefore necessary to identify younger sources, that are expected to accelerate to higher energies with flat spectra. Future instruments like CTA and HAWC, in combination with multiwavelength data from e.g. electron synchrotron radiation and ionization signatures, will help to further pin-point SNRs as CR sources, or even to point towards a different scenario. In particular, these two instruments will be able to provide data up to $100-300 \mathrm{TeV}$ gamma-ray energy, corresponding to $1-3 \mathrm{PeV} \mathrm{CR}$ energy. This way, Galactic PeVatrons can be identified and by this those sources contributing to the CR spectrum in the knee region.

- The first detection of astrophysical high-energy neutrinos provides a new window to $\mathrm{CR}$ physics. While both the spatially isotropic distribution of the signal, the measurement up to $2 \mathrm{PeV}$ neutrino energies as well as the intensity of the signal point to a mainly extragalactic origin of the signal, a fraction of the events can certainly be of Galactic origin. The detailed knowledge from diffuse and local gammaray measurements help to narrow down the maximum contribution to 2-3 events for the current analysis. With future instruments like KM3NeT and IceCubeGen2, the sensitivity for point source searches will be drastically increased. It is therefore expected that neutrino astronomy with next generation's telescopes will contribute significantly to identifying the sources of Galactic CRs or at least exclude certain standard scenarios.

This work was supported by the DFG research unit FOR1048: Instabilities, Turbulence and Transport in Cosmic Magnetic Fields. Support is also acknowledged from the Ruhr AstroparticlePlasma Physics Center (RAPP Center), Bochum and from the Research Department: Plasmas with Complex Interactions, Bochum.

\section{References}

[1] F. Salmida et al. (2011), International Cosmic Ray Conference, arXiv: 1107.4809

[2] W. Baade, F. Zwicky, Proc. Nat. Acad. Sci. 20, 259 (1934)

[3] L. O'C. Drury, Invited talk at CRISM2014 (2014), arXiv: 1412.1376

[4] R. Diehl et al., Nature 439, 45 (2006)

[5] J.K. Becker, Phys. Rep. 458, 173 (2008)

[6] E. Fermi, Phys. Rev. 75, 1169 (1949)

[7] E. Fermi, ApJ 119, 1 (1954)

[8] A.R. Bell, MNRAS 182, 147 \& 443 (1978)

[9] R. Schlickeiser, ApJ 336, 243 \& 264 (1989)

[10] A.R. Bell, MNRAS 353, 550 (2004)

[11] J. Vink, A\&A Rev. 20, 49 (2012), 1112.0576

[12] K.M. Schure, A.R. Bell, MNRAS 435, 1174 (2013)

[13] P.L. Biermann et al., PRL 103, 061101 (2009)

[14] P.L. Biermann et al., ApJ 725, 184 (2010)

[15] P.L. Biermann et al., ApJ 710, L53 (2010)

[16] H.S. Ahn et al., ApJ 714, L89 (2010)

[17] V. Choutko et al. (2013), International Cosmic Ray Conference

[18] O. Adriani et al., Science 332, 69 (2011)

[19] S. Haino et al. (2013), International Cosmic Ray Conference

[20] S. Thoudam, J.R. Hörandel, MNRAS 435, 2532 (2013)

[21] P. Blasi, A\&A Rev. 21, 70 (2013), 1311.7346

[22] W.D. Apel et al., Astrop. Phys. 47, 54 (2013)

[23] W.D. Apel et al., Adv. in Space Res. 53, 1456 (2014)

[24] J.R. Hörandel, Astrop. Phys. 21, 241 (2004)

[25] D. Allard, E. Parizot, A.V. Olinto, Astrop. Phys. 27, 61 (2007)

[26] D. García-Gámez et al. (2013), International Cosmic Ray Conference, arXiv: 1307.5059

[27] R.U. Abbasi et al., Astrop. Phys. 64, 49 (2015)

[28] T.K. Gaisser, T. Stanev, S. Tilav, Frontiers of Physics 8, 748 (2013)

[29] V. Berezinsky, Astrop. Phys. 53, 120 (2014), 1301.0914 
[30] M. Tluczykont et al., Astrop. Phys. 56, 42 (2014), 1403.5688

[31] G. Di Sciascio, R. Iuppa (Nova Science Publishers, 2013), arXiv1407.2144

[32] M. Pohl, D. Eichler, ApJ 766, 4 (2013)

[33] C. Evoli et al., PRL 108, 211102 (2012)

[34] P.L. Biermann et al., ApJ 768, 124 (2013)

[35] A. Lazarian, P. Desiati, ApJ 722, 188 (2010)

[36] G. Giacinti, G. Sigl, PRL 109, 071101 (2012)

[37] M.M. Romanova et al., ApJ 630, 1020 (2005)

[38] K. Mannheim, R. Schlickeiser, A\&A 286, 983 (1994)

[39] M. Aguilar et al., PRL 113, 121102 (2014)

[40] L. Bergström, T. Bringmann, J. Edsjö, PRD 78, 103520 (2008)

[41] P. Mertsch, S. Sarkar, PRD 90, 061301 (2014)

[42] W.L. Kraushaar, G.W. Clark, PRL 8, 106 (1962)

[43] E. Lorenz, R. Wagner, EPJ H 37, 459 (2012)

[44] F.M. Rieger, E. de Oña-Wilhelmi, F.A. Aharonian, Frontiers of Physics 8, 714 (2013)

[45] G.R. Blumenthal, R.J. Gould, Reviews of Modern Physics 42, 237 (1970)

[46] S.R. Kelner, F.A. Aharonian, V.V. Bugayov, PRD 74, 034018 (2006)

[47] M. Mandelartz, J. Becker Tjus, Astrop. Phys. 65, 80 (2015)

[48] N. Nierstenhöfer et al., Galactic Propagation of Cosmic Rays from Individual Supernova Remnants, Proceedings to be published IOP conf. ser. (2014)

[49] A.W. Strong, I.V. Moskalenko, ApJ 509, 212 (1998)

[50] C. Evoli et al., JCAP 10, 018 (2008)

[51] R. Kissmann, Astrop. Phys. 55, 37 (2014), 1401.4035

[52] A.A. Abdo et al., Science 327, 1103 (2010)

[53] M. Ackermann et al., Science 339, 807 (2013)

[54] T. Jogler et al. (2015), SuGAR, Geneva, Switzerland, http://indico.cern.ch/event/332221/

[55] B.S. Acharya et al., Astrop. Phys. 62, 152 (2015)

[56] J.K. Becker et al., ApJ 739, L43 (2011)
[57] F. Schuppan et al., A\&A 541, A126 (2012)

[58] N. Indriolo et al., ApJ 724, 1357 (2010)

[59] S. Vaupré et al., A\&A 568, A50 (2014), 1407.0205

[60] F. Schuppan, C. Röken, J. Becker Tjus, A\&A 567, A50 (2014)

[61] B.S. Acharya et al., Astrop. Phys. 43, 3 (2013)

[62] A.U. Abeysekara et al., Astrop. Phys. 50, 26 (2013)

[63] E. Waxman, J.N. Bahcall, PRD 59, 23002 (1999)

[64] M.D. Kistler, J.F. Beacom, PRD 74, 063007 (2006)

[65] M.G. Aartsen et al., Science 342, 1242856 (2013)

[66] M.G. Aartsen et al., PRL 113, 101101 (2014)

[67] M.G. Aartsen et al., PRD 91, 022001 (2015)

[68] A. Neronov, D. Semikoz, C. Tchernin, PRD 89, 103002 (2014)

[69] J.C. Joshi, W. Winter, N. Gupta, MNRAS 439, 3414 (2014)

[70] A. Fedynitch, J. Becker Tjus, P. Desiati, PRD 86, 114024 (2012)

[71] F. Scheriau, IceCube Collaboration, Unfolding of the Muon Neutrino Energy Spectrum with IceCube, Proceedings to be published IOP conf. series (2014)

[72] M. Schmitz, IceCube Collaboration, Unfolding of the Muon Neutrino Energy Spectrum with IceCube, Poster presentation at Neutrino 2014, Boston (2014)

[73] J. Becker Tjus et al., PRD 89, 123005 (2014)

[74] K. Murase, M. Ahlers, B.C. Lacki, PRD 88, 121301 (2013)

[75] J.K. Becker et al., Astrop. Phys. 28, 98 (2007)

[76] M.G. Aartsen et al., arXiv:1406.6757 (2014), Accepted for publication in ApJ

[77] S. Adrián-Martínez et al., ApJ 786, L5 (2014)

[78] KM3NeT, Technical Design Report for a Deep-Sea Research Infrastructure in the Mediterranean Sea Incorporating a Very Large Volume Neutrino Telescope (2014), http://www. km3net.org/TDR/ TDRKM3NeT.pdf

[79] M.G. Aartsen et al., arXiv: 1412.5106 (2014) 